\title{
EXTENSIVE PROLIFERATION OF CD4+ LYMPHOCYTE BY BOTH PHYTOHAEMAGGLUTININ A AND ANTI-CD2/CD3/ CD28 MACSIBEADS
}

\author{
M. Mirzakhani ${ }^{1}$, M. Shahbazi ${ }^{2}$, S. Darvish ${ }^{1}$, M. Mohammadnia-Afrouzi ${ }^{2}$ \\ ${ }^{1}$ Student Research Committee, School of Medicine, Babol University of Medical Sciences, Babol, I.R.Iran \\ ${ }^{2}$ Department of Immunology, School of Medicine, Babol University of Medical Sciences, Babol, I.R.Iran
}

\begin{abstract}
Background: Lymphocytes proliferate considerably following appropriate stimulation in vitro. Autologous $T$ cells are obtained from whole blood or tissue sites in relatively limited amounts. We need a method to expand these cells efficiently, study their functions and manipulate them to create appropriate cells for transferring to the patient with infection and cancer. Objectives: The aim of this study is to determine proliferation ability of two different stimulators on CD4+ lymphocytes. Methods: Lymphocytes were isolated from blood samples of healthy donors after removing adherent cells (monocytes). The efficacy of MACSiBead ${ }^{\mathrm{TM}}$ coated with anti-CD2, anti-CD3, anti-CD28 (anti-CD2/CD3/CD28) was compared with Phytohaemagglutinin A (PHA) on CD4+ lymphocytes proliferation using carboxyfluorescein diacetate succinimidyl ester (CFSE) in cell culture media. The percentage of proliferating cells was analyzed using flow cytometry. Results: Both stimulators induced extensive proliferation of CD4+ lymphocytes but proliferation ability of PHA was higher compared to stimulation by anti-CD2/CD3/CD28 MACSiBead ${ }^{\mathrm{TM}}$. The proliferation rate of cells stimulated by PHA was $93.8 \% \pm 3.37 \%$ whereas it was $85.2 \% \pm 4.7 \%$ in cells stimulated by anti-CD2/CD3/CD28 MACSiBead ${ }^{\mathrm{TM}}$. Conclusions: Our results show that MACSiBead ${ }^{\mathrm{TM}}$ along with PHA can be used to obtain a large number of expanded CD4+ lymphocytes.
\end{abstract}

Key words: anti-CD2/CD3/CD28 MACSiBead ${ }^{\mathrm{TM}}$, PHA, proliferation

Corresponding author: Mousa Mohammadnia-Afrouzi, Department of Immunology, School of Medicine, Babol University of Medical Sciences, Ganj Afrooz Avenue, Babol, Mazandaran, I.R.Iran 47176-47745. e-mail: m.mohammadnia@mubabol.ac.ir., tel: +989112151198, fax: +98 1132190181

\section{INTRODUCTION}

$\mathrm{n}$ vitro Lymphocyte's stimulation methods are extensively used for expansion of these cells, adoptive immunotherapy of cancer and study of their function [1, 2]. PHA as a mitogenic molecule and monoclonal antibodies yield polyclonal proliferation whereas specific antigen promotes a monoclonal or oligoclonal response. PHA activates lymphocytes through binding to cell membrane glycoproteins such as the T-cell receptor (TCR)-CD3 complex. There are a number of mitogenic antibodies containing those engaged towards the TCR, CD2, CD3 and CD28 molecule [1]. Specific antibodies against TCR- 
CD3 complex provide an initial activation signal, but proliferation is dependent on a co-stimulatory signal, mostly provided by means of the CD28 molecule [1, 3-5]. These antibodies are often bound to accessory cells, beads, or a solid surface to mimic professional APCs $[1,6]$ because free antibodies usually provide insufficient cross-linking of the receptor to activate intracellular signaling cascades [1].

With success of $T$ cells manipulation and stimulation methods [4] such as PHA, autologous antigen presenting cells with specific peptide, and anti-CD3 and anti-CD28-coated beads [7], it is possible to stimulate and expand lymphocytes to adoptive transfer $[4,7]$. Nowadays, adoptive transfer of $T$ cells is known successful treatment for infections and cancer such as HIV infection and multiple myeloma [3, 8-10]. For adoptive transferring of $T$ lymphocyte, a large number of cells are required, and on the other hand, efficiently, adoptive transfer need an antigenspecific $T$ cell obtaining from blood or tissue sites, but the obtained cells are not sufficient [4]. The abovementioned methods were usually used to efficiently expand these cells for the next investigation [11]. The aim of this study is to define proliferation ability of two different stimulators on CD4+ lymphocytes.

\section{OBJECTIVES}

PHA and anti-CD2/CD3/CD28 MACSiBead ${ }^{\mathrm{TM}}$ were used to define which of them cause more proliferated-CD4+ lymphocytes.

\section{MATERIALS AND METHODS}

\section{Isolation of lymphocytes from peripheral blood}

Three healthy individuals were enrolled in this study. Freshly peripheral blood samples were collected from the healthy donors under written informed consent. Peripheral blood mononuclear cells (PBMCs) were separated using Ficoll-Paque density gradient centrifugation (Biowest, France). To prepare lymphocytes for selected experiments, PBMCs were incubated in a cell culture flask in 5\% CO2 incubator in order to remove adherent cells (monocytes). Lymphocytes were isolated from PBMCs after removing adherent cells (monocytes) then CD4+ $\mathrm{T}$ lymphocytes were isolated and expanded as described below.

\section{CD4+ $T$ lymphocytes isolation \& Culture}

CD4+ cells were enriched with an isolation kit containing a cocktail of CD8, CD14, CD16, CD19, CD36, CD56, CD123, TCR $/ \delta$, and CD235a (glycophorin A) (MACS, Miltenyi Biotec, Bergisch Gladbach, Germany). Then, CD4+ cells were purified using APC labeled anti-CD4 antibodies (Ebioscience, USA). The purity of isolated CD4+ $\mathrm{T}$ lymphocytes was above $87 \%$ based on CD4 expression as assessed by flow cytometry. The CD4+ T lymphocytes were labeled with carboxyfluorescein diacetate succinimidyl ester (CFSE) (Invitrogen, Life Technologies, Carlsbad, CA), which is used to examine cells division and expansion after stimulation. Briefly, to label cells, $1 \times 10^{6}$ lymphocytes maintained in RPMI 1640 containing $10 \%$ fetal calf serum (RPMI/FCS) were incubated with $2 \mu \mathrm{M} \mathrm{CFSE}$ at $37^{\circ} \mathrm{C}$ for $10 \mathrm{~min}$. Lymphocytes were then washed two times to remove unbound CFSE, resuspended in a fresh medium. The CFSE-labeled cells were cultured in RPMI-1640 medium (Gibco, USA) containing $10 \%$ fetal calf serum (Biosera, France), $100 \mathrm{U} / \mathrm{mL}$ penicillin/100 $\mu \mathrm{g} /$ $\mathrm{mL}$ streptomycin (Gibco, USA), $2 \mathrm{~mm}$ l-glutamine (Gibco, USA), $1 \mathrm{~mm}$ sodium pyruvate (Gibco, USA) supplemented with PHA (Gibco, USA) or anti-CD2/ CD3/CD28 MACSiBead ${ }^{\mathrm{TM}}$ (Miltenyi Biotech, Germany). Labeled cells were then distributed $(100,000 /$ well) in a 96 well round bottom plate in the presence of anti-CD2/CD3/CD28 MACSiBead ${ }^{\mathrm{TM}}$ (three beads/ cell), PHA or no additional stimulator and were fed with the further fresh medium at day 3 .

Monitoring CD4+ lymphocyte cells division and expansion using CFSE

The expansion after stimulation was analyzed using methods described following. Cells were analyzed on FACSCalibur flow cytometry (Becton Dickinson, USA) at day 5. The proportion of CD4+ gated lymphocytes undergoing 0-6 divisions then were quantitated based on the pattern of CFSE fluorescence using Flow-Jo software.

\section{Statistical analysis}

The data were analyzed by the Mann-Whitney $U$ test with the use of GraphPad Prism software for Windows (GraphPad Software, La Jolla, CA, USA). Data were presented as means $\pm S D$. Differences were considered significant at $p$ value $<0.05$.

\section{RESULTS}

To determine the effect of anti-CD2/CD3/CD28 MAC$\mathrm{SiBead}^{\mathrm{TM}}$ and PHA on the expansion and proliferation ability of human CD4+ T lymphocytes, we cultured isolated Lymphocytes from healthy individuals in different conditions. First, $1 \times 10^{6}$ CFSE labeled lymphocytes were cultured and stimulated with antiCD2/CD3/CD28 MACSiBead ${ }^{\mathrm{TM}}$ and PHA. To set the undivided peak fluorescence, CFSE labeled lymphocytes were also cultured in the absence of any stimulators (untreated CD4+ lymphocytes in fig- 
ures). Lymphocytes were harvested and stained for CD4 marker, and then analyzed for CFSE using flow cytometry. A CFSE division profile of anti-CD2/CD3/ CD28 MACSiBead ${ }^{\mathrm{TM}}$ and PHA stimulated CD4+ lymphocyte during six days are illustrated in following figures (fig. 1 A, B, C). Up to five or six separate division cycles could be visualized by different CFSE signal peaks. More cell divisions were difficult to determine as peaks tended to be compact.

As shown in figure 1, the results indicate a difference in the proliferation ability of PHA and anti-CD2/CD3/
CD28 MACSiBead ${ }^{\mathrm{TM}}$, although each of these stimulus methods resulted in extensive CD4+ lymphocyte proliferation. The Proliferation of CD4+ Iymphocytes stimulated by PHA showed an increase when compared with the cells stimulated by anti-CD2/CD3/ CD28 MACSiBead ${ }^{\mathrm{TM}}$. The proliferation rate of cells stimulated by PHA was $93.8 \% \pm 3.37 \%$, whereas it was $85.2 \% \pm 4.7 \%$ in cells stimulated by anti-CD2/ CD3/CD28 MACSiBead ${ }^{\mathrm{TM}} \mathrm{s}$. There was no significant difference in the proliferation ability of PHA and antiCD2/CD3/CD28 MACSiBead ${ }^{\mathrm{TM}}$ ( $p$ value $=0.1$ ).
A)

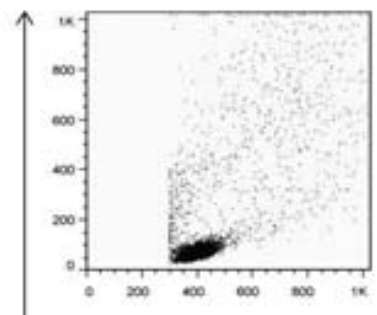

B)

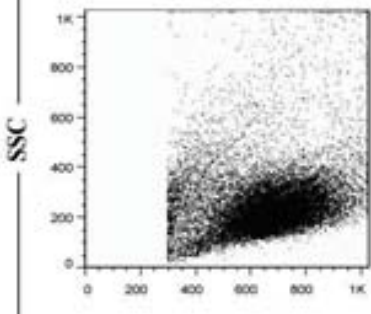

C)
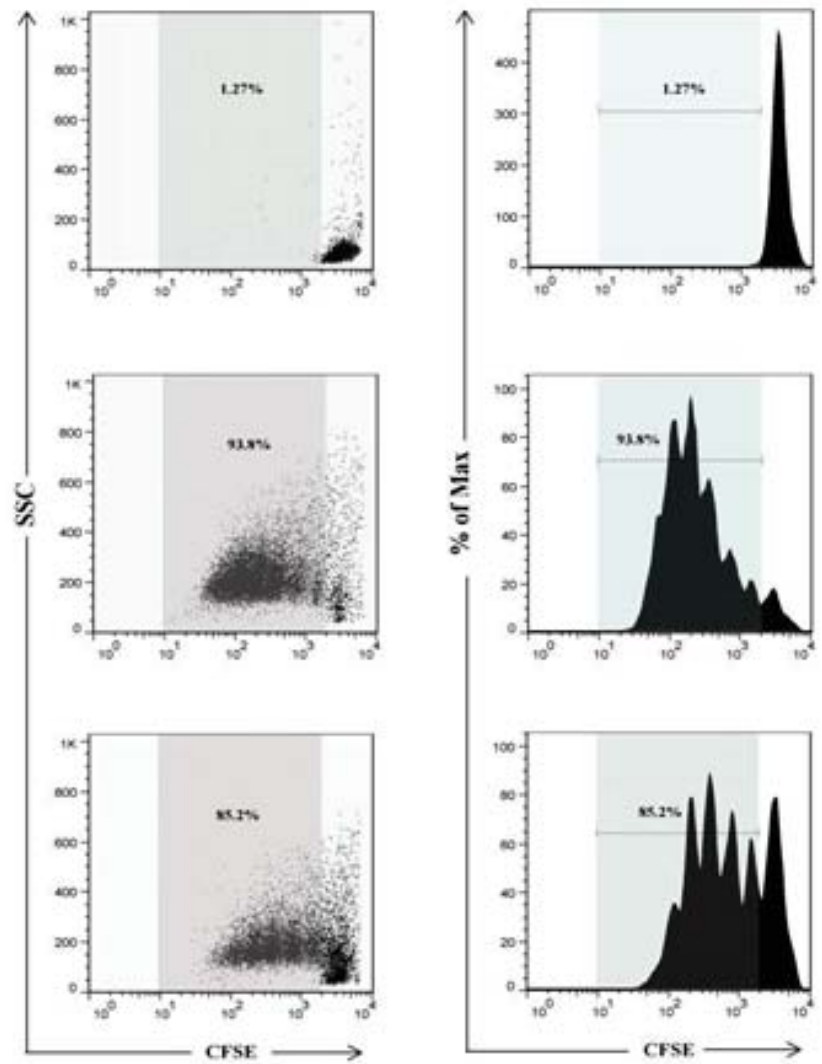

Fig. 1. (A) Flow cytometric dot plot for forward scatter versus side scatter on purified CFSE- labeled untreated CD4+ T lymphocytes have been shown. The cells then were analyzed for CFSE to monitor cells division and expansion. (B) Flow cytometric dot plot for forward scatter versus side scatter on purified CFSE - labeled, PHA-treated CD4+ T lymphocytes has been indicated. The cells were undergoing 0-6 divisions then were analyzed based on the pattern of CFSE fluorescence. (C) Flow cytometric dot plot for forward scatter versus side scatter on purified CFSE-labeled anti-CD2/CD3/CD28 MACSiBead ${ }^{\mathrm{TM}}$-treated CD4+ T lymphocytes have been shown. The cells undergoing 0-6 divisions then were analyzed for CFSE, and the rate of proliferation has been indicated.

\section{DISCUSSION}

Antigen-specific CD4+ T lymphocytes are produced by adding repeated cycles of the stimulating antigen with autologous antigen-presenting cells and IL-2. We can reduce the requirement for large amounts of specific antigen by adding anti-CD2/CD3/CD28 MACSiBead $^{\mathrm{TM}}$ after the primary antigen stimulation, without loss of antigen specificity [1].

As the adoptive transfer of autologous $T$ cells is useful for patients with infections and cancer $[3,8]$, we analyzed proliferation ability of the two methods including anti-CD3, anti-CD2, anti-CD28-coated beads, and PHA. Many studies used anti-CD3/CD28 coated beads but not anti-CD3, anti-CD2 and antiCD28-coated beads to expand T cells $[1,3,8,12]$. In the current study, in addition to anti-CD3/CD28, anti-CD2 was used in order to increase the signals received by $T$ cells which improve the stimulation and enhance the proliferation of T cells. Our results indicated that the proliferation ability of the PHA method was higher than anti-CD3, anti-CD2, and anti-CD28- 
coated beads method. However, with considering the rate of proliferation ability of MACSiBead ${ }^{\mathrm{TM}}$ and $\mathrm{PHA}$ which was $85.2 \%$ and $93.8 \%$, respectively, it can be said that MACSiBead ${ }^{\mathrm{TM}}$ along with PHA provides a simple, fast and reproducible method for polyclonal $\mathrm{T}$ cell stimulation and yield a large number of expanded CD4+ lymphocytes.

Conclusion: Finally, expanded $T$ cells can be used in $T$ cell functional assays either directly after stimulation or after extensive proliferation [1]. In addition, $T$ cells can be manipulated to improve their specificity and increase their robust responsiveness for the next investigations.

\section{Acknowledgement}

This work was financially supported by a research grant (No. 4948) from Babol University of Medical Sciences.

\section{REFERENCES}

1. Trickett $A, K$ wan $Y L$. T cell stimulation and expansion using anti-CD3/CD28 beads. J Immunol Methods, 275, 2003, 251-5.

2. Lamers $C$, Van de Griend R, Braakman E, et al. Optimization of culture conditions for activation and large-scale expansion of human T lymphocytes for bispecific antibody-directed cellular immunotherapy. Int J Cancer, 51, 1992, 973-9.

3. Kalamasz D, Long S, Taniguchi R, et al. Optimization of human T-cell expansion ex vivo using magnetic beads conju- gated with anti-CD3 and Anti-CD28 antibodies. J Immunother, 27, 2004, 405-18.

4. Li Y, Kurlander RJ. Comparison of anti-CD3 and anti-CD28coated beads with soluble anti-CD3 for expanding human $T$ cells: differing impact on CD8 T cell phenotype and responsiveness to restimulation. J Transl Med, 8, 2010, 104.

5. Teschner D, Wenzel G, Distler E, et al. In vitro stimulation and expansion of human tumour-reactive CD8+ cytotoxic T lymphocytes by anti-CD3/CD28/CD137 magnetic beads. Scand J Immunol, 74, 2011, 155-64.

6. Martkamchan S, Onlamoon N, Wang S, et al. The Effects of Anti-CD3/CD28 Coated Beads and IL-2 on Expanded T Cell for Immunotherapy. Adv Clin Exp Med, 25, 2016, 821-8.

7. Onlamoon N, Boonchan M, Unpol P, et al. Influence of cell isolation method on the optimization of CD4+ $\mathrm{T}$ cell expansion using anti-CD3/CD28 coated beads, Asian Pac J Allergy Immunol. 31, 2013, 99.

8. Bernstein WB, Cox JH, Aronson NE, et al. Immune reconstitution following autologous transfers of CD3/CD28 stimulated CD4+ T cells to HIV-infected persons. Clin Immunol, 111, 2004, 262-74.

9. Trickett $A E$, Kwan $Y L$, Cameron $B$, et al. Ex vivo expansion of functional $\mathrm{T}$ lymphocytes from HIV-infected individuals. J Immunol Methods, 262, 2002, 71-83.

10. Thomas AK, Maus MV, Shalaby WS, et al. A cell-based artificial antigen-presenting cell coated with anti-CD3 and CD28 antibodies enables rapid expansion and long-term growth of CD4 T lymphocytes. Clin Immunol (Orlando, Fla), 105, 2002, 259-72.

11. Röth $A$, Schneider $L$, Himmelreich $H$, et al. Impact of culture conditions on the proliferative lifespan of human T cells in vitro. Cytotherapy, 9, 2007, 91-8.

12. Onlamoon N, Plagman N, Rogers KA, et al. Anti-CD3/28 mediated expansion of macaque CD4+ T cells is polyclonal and provides extended survival after adoptive transfer, J Med Primatol, 36, 2007, 206-18. 